\title{
A Percepção dos Gestores Acerca do Desempenho de Trabalhadores com Diferentes Tipos de Deficiência
}

\author{
Janayna de Cássia Coelho Suzano \\ Maria Nivalda de Carvalho-Freitas* \\ Raissa Pedrosa Gomes Tette \\ Carla Regina Guimarães Brighenti \\ Marcos Vieira-Silva \\ Universidade Federal de São João Del Rei, São João Del Rei, Minas Gerais, Brasil
}

\begin{abstract}
RESUMO
Esta pesquisa pretende contribuir para o entendimento do processo de inserção de pessoas com deficiência (PcDs) no trabalho. O principal objetivo foi verificar se os gestores avaliam o desempenho das PcDs diferentemente, de acordo com o tipo de deficiência que possuem. Realizou-se um estudo descritivo e exploratório com amostra de 123 gestores de PcDs, que atuam em 14 empresas localizadas no Espírito Santo/Brasil. Os resultados mostraram que diferentes critérios de avaliação de desempenho são utilizados pelos gestores, por tipo de deficiência. Porém, na análise qualitativa, os gestores, quando atuavam diretamente com pessoas de diferentes tipos de deficiência, evidenciaram a existência de similaridades na avaliação do desempenho dessas pessoas.
\end{abstract}

Palavras-chave: pessoas com deficiência; gestão; análise de desempenho; inclusão; mercado de trabalho.

\section{ABSTRACT \\ The Perception of Managers on the Performance of Employees With Different Types of Disabilities}

This research intends to contribute to understanding about the process of insertion of people with disability (PwDs) in the workplace. The main objective was to verify whether the managers have different ways of seeing people with disabilities, considering the type of disability. It was conducted a cross-sectional descriptive study, with a sample of 123 managers of PwDs, working in 14 companies located in Espírito Santo/Brazil. The results showed that managers see the PwD differently, depending on the type of disability. However, in qualitative analysis, the managers demonstrated the existence of similarities in the assessment of performance of these people.

Keywords: people with disability; management; performance evaluation; inclusion; labor market.

Um levantamento mundial constatou que aproximadamente $10 \%$ da população do mundo (650 milhões de pessoas) possui algum tipo de deficiência, sendo que $72 \%$ delas estão em idade laboral (Organização Internacional do Trabalho, 2009). No Brasil, segundo dados do censo realizado em 2010 pelo Instituto Brasileiro de Geografia e Estatística (IBGE, 2000), existem 45,6 milhões de pessoas com pelo menos um tipo de deficiência, o que representa $23,9 \%$ da população nacional.

Esse percentual é distribuído da seguinte forma: a deficiência visual apresentou a maior ocorrência, afetando $18,6 \%$ da população brasileira; em segundo lugar está a deficiência física, ocorrendo em $7 \%$ da população; seguida da deficiência auditiva, em 5,10\%; 
e da deficiência mental ${ }^{1}$ ou intelectual, em 1,40\%. Esses dados indicam que, no Brasil, o maior número de pessoas com deficiência (PcDs) possui deficiências visual (não necessariamente cegueira total) e física, respectivamente; e a deficiência intelectual é a de menor proporção na população (Cartilha do Censo 2010, 2012).

As pessoas com deficiência são amparadas no âmbito profissional pela Constituição Federal, por meio da política de cotas expressa na Lei n. 8.213 (1991) e do Decreto n. 3.298 (1999), que destina a elas um percentual de reserva de cargos, variável de acordo com o número de empregados das instituições que possuem 100 ou mais trabalhadores em seu quadro. Nesse sentido, à pessoa com deficiência é assegurado o acesso legal ao mercado formal de trabalho.

No entanto, apesar do amparo constitucional, a participação das pessoas com deficiência no mercado de trabalho ainda é baixa quando comparada a das pessoas sem deficiência. Em 2010, a população ocupada com pelo menos uma das deficiências investigadas era de $23,6 \%$ do total de ocupados. A população sem deficiência empregada era de $76,4 \%$ do total (Cartilha do Censo 2010, 2012).

Devido à fiscalização do Ministério Público do Trabalho, embasada na Lei de Cotas (Lei 8.213/91), que prevê o pagamento de multa às empresas que não cumprem a Lei, houve um aumento da participação das pessoas com deficiência no mercado formal de trabalho, conforme indica um levantamento realizado pela Relação Anual de Informações Sociais (RAIS) (MTE, 2012).

De acordo com o levantamento da RAIS (MTE, 2012), em 2012 verificou-se um aumento de 1,54\% no número de pessoas declaradas como PcDs, representando um acréscimo de cinco mil empregos. Com esse resultado, somam-se 330,3 mil vínculos declarados, o que corresponde a $0,70 \%$ do total dos vínculos empregatícios, sinalizando uma relativa estabilidade, quando comparado com o resultado registrado em 2011 (0,70\%). Do total de 330,3 mil vínculos ativos, 214,7 mil eram do gênero masculino e 115,6 mil do feminino, o que indica uma proporção de $65 \%$ do total de homens e de $35 \%$ para mulheres com deficiência, demonstrando uma ligeira queda da participação dos homens, quando comparado com os resultados de $2011(65,74 \%)$.
Pesquisas indicam que há falta de preparo social, assim como inúmeras barreiras funcionais e arquitetônicas nas organizações nacionais que dificultam o processo de inserção da PcD (Batista, 2004; CarvalhoFreitas, 2007; Nohara, Acevedo \& Fiametti, 2009). Conforme Tanaka e Manzini (2005), ao se contratar uma pessoa com deficiência com o único intuito de cumprir a Lei, sem propiciar adaptações que facilitem o acesso dessa pessoa, pode-se contribuir para exacerbar o preconceito de que ela não possui competência laboral.

No entanto, existem poucos estudos a respeito do contexto brasileiro que abordam a gestão do trabalho das PcDs já inseridas na organização (Alves \& Galeão-Silva, 2004; Carvalho-Freitas \& Marques, 2007; Heinski, 2004; Ribeiro \& Ribeiro, 2009), e nenhum deles estudou a gestão das PcDs analisando as diferenças desse processo no ambiente de trabalho de acordo com cada tipo de deficiência.

Carvalho-Freitas, Souto, Simas, Costa e dos Santos (2012) realizaram uma pesquisa com universitários visando verificar a disposição de futuros profissionais para trabalhar com PcDs. Foi constatado que essas disposições estavam associadas à percepção de dificuldades para inserir pessoas com deficiência, como falta de conhecimento sobre recursos materiais, tecnológicos e de processos para garantir a acessibilidade dos espaços físicos, além de falta de convivência com as pessoas com deficiência. Esses resultados reforçam a necessidade de orientar ações que modifiquem as condições e políticas organizacionais, visando possibilitar maior contato entre pessoas com e sem deficiência.

Inúmeras pesquisas internacionais têm, desde o trabalho de Allport (1954), identificado a relevância do contato intergrupos para minimização do preconceito (Pettigrew, 1997; Pettigrew \& Tropp, 2006; Pettigrew et al., 2011). Essas pesquisas, que inicialmente focavam nas relações entre grupos culturais e raciais diferentes, passaram também a contemplar outros grupos estigmatizados como idosos, pessoas com deficiência, doentes mentais e homossexuais (Pettigrew et al., 2011). Allport (1954) defendeu a premissa de que o contato intergrupal para reduzir atitudes negativas precisava atender quatro condições: (a) status igual entre os grupos na situação; (b) objetivos comuns; (c) não competição entre os grupos; e (d) sanção legal para os contatos. Pesquisas mais recentes 
têm indicado que essas condições facilitam a relação, embora não seja estritamente necessário a presença de todas elas (Pettigrew, 1997; Pettigrew \& Tropp, 2006).

Especificamente sobre pessoas com deficiência, objeto desta investigação, as pesquisas nacionais e internacionais têm indicado que, principalmente, o status igual intergrupos não é atendido nas relações com essas pessoas, especialmente em situações de trabalho. Conforme Trice e Beyer (1993), nas organizações capitalistas de trabalho alguns valores são enfatizados: a racionalidade e a despersonalização no processo de decisão; a competitividade; o individualismo e a autossuficiência; a alocação dos resultados baseada na proporcionalidade; a eficiência; e a padronização no desempenho das tarefas. Stone-Romero, Stone e Lukaszewski (2006) afirmam que, nesse contexto apresentado, as PcDs são vistas como: incapazes para competir ou alcançar os padrões de performance requeridos; dependentes de outras; incapazes de garantir a performance a menos que lhes sejam garantidas condições especiais de trabalho; não se enquadram no protótipo valorizado pela sociedade (jovem, do sexo masculino, atraente e fisicamente em forma); e são vistas como tendo menor status social que as demais pessoas, o que dificulta a inclusão delas no trabalho. Além disso, pesquisas indicam que as PcDs são normalmente objeto de piedade e do estereótipo de incompetência, reforçando comportamentos de superproteção e consequente menor status do grupo (Cuddy, Fiske, \& Glick, 2007).

Além disso, as pesquisas também indicam que estas costumam ser desfavorecidas na avaliação de seu desempenho. Conforme Jones, Latreille e Sloane (2006), quando um trabalhador tem deficiência, os gestores podem ter baixas expectativas sobre seu desempenho, as quais são baseadas na relação entre a deficiência e a capacidade da $\mathrm{PcD}$ para atender às expectativas de determinada função. Por outro lado, Stone-Romero, Stone e Lukaszewski (2006) sugerem que os gestores têm alto grau de exigência do desempenho das PcDs, e que se estas não atenderem completamente às expectativas do seu superior, terão sua performance considerada negativa.

Segundo Nascimento, Damasceno e Assis (2008), as métricas utilizadas por alguns gestores para avaliar o desempenho dos empregados são perversas, uma vez que a avaliação é realizada com base em um desempenho idealizado. Assim, mede-se o desempenho dos melhores trabalhadores (mais produtivos) em condições ideais (descansados, motivados) e dispondo de todos os recursos que precisam para executar suas atividades. Essas métricas são generalizadas para a análise do desempenho de uma PcD, a qual, muitas vezes, não usufrui de um ambiente adaptado para a execução de sua atividade.

A avaliação de desempenho, como um dos elementos da gestão estratégica de pessoas e, consequentemente, da gestão da diversidade, é um importante processo a ser analisado com vistas a compreender e potencializar a inserção de PcDs no mercado de trabalho. Atualmente, o modelo mais empregado é o de gestão estratégica de recursos humanos, que, como o próprio nome pressupõe, considera a gestão de pessoas como parte integrante da estratégia organizacional. Nesse modelo, o desempenho do trabalhador contempla muito mais do que a pura execução de sua atividade. A avaliação de desempenho considera, então, o trabalho executado em nível individual e sua implicação para os níveis micro e macro-organizacionais (Fleury \& Fleury, 2001).

Nessa perspectiva, conforme Brandão e Guimarães (2001), a avaliação do desempenho é um dos processos da gestão de desempenho que avalia o trabalho executado pelo trabalhador de acordo com os processos, objetivos e metas empresariais. O gestor deve considerar o trabalhador e o seu trabalho dentro de um contexto organizacional e social amplo.

Dessa maneira, ao se pensar na gestão da diversidade e, mais especificamente, na gestão das PcDs, é necessário contemplar a adaptação das condições e práticas de trabalho, uma vez que a existência ou a carência de adaptações norteiam a relação entre as pessoas nas organizações e interferem no desempenho das PcDs, pois elas poderão ser mais autônomas e eficientes em um ambiente propício para realização do trabalho (Carvalho-Freitas, 2007).

Sassaki (1999) aponta que a inclusão das PcDs abarca a noção de mudanças sociais que permitam a plena autonomia e cidadania. Nesse sentido, o ambiente de trabalho deve ser reestruturado de maneira a eliminar tanto as barreiras físicas - arquitetônicas e de mobiliários, que dificultam a mobilidade autônoma - 
quanto as de comunicação e as atitudinais (discriminação, preconceito e estigmas), de modo a ser acessível a todos. Enquanto Wilson-Kovacs, Ryan, Haslam e Rabinovich (2008) discutem como as diversas barreiras organizacionais influenciam na progressão na carreira das PcDs, sendo elas um elemento dificultador para ascensão dessas pessoas a posições de liderança. Zolna, Sanford, Sabata e Goldthwaite (2007) e Björk (2009) exploram como o ambiente físico, abarcando as tecnologias assistivas, está relacionado à probabilidade de entrada e permanência das PcDs no trabalho.

Carneiro e Ribeiro (2008) observaram que as empresas buscam contratar pessoas com deficiência que não requerem adaptações no ambiente e que não possam significar diminuição de produtividade. Por isso, têm prioridade as pessoas com deficiência física parcial, não-cadeirantes e as pessoas com deficiência auditiva leve. O estudo de Goulart e Coimbra (2008) reafirma a propensão dos gestores para contratação de pessoas com deficiência física ou com deficiência auditiva. Dos 228 trabalhadores com deficiência de uma empresa de reflorestamento, 137 tinham deficiência física e 41 , deficiência auditiva. O número de trabalhadores com deficiências intelectual e múltipla é pequeno (14 e 10 trabalhadores, respectivamente). Pesquisa realizada por Batista (2004) em empresas do estado de Minas Gerais também corrobora essa tendência ao verificar que $37 \%$ das pessoas com deficiência contratadas tinham deficiência auditiva; $26 \%$, deficiência física; $17 \%$, deficiência múltipla; somente $7 \%$, visual; e, por fim, um percentual muito baixo de pessoas com deficiência intelectual: apenas $3 \%$.

O Instituto Ethos (2002) também desenvolveu uma pesquisa nas 500 maiores empresas do Brasil e os resultados apontaram que, enquanto no quadro funcional somente $1,5 \%$ dos trabalhadores têm deficiência (0,8\% têm deficiência física, $0,1 \%$ têm deficiência visual, $0,5 \%$ têm deficiência auditiva e $0,1 \%$ têm deficiência múltipla), no quadro de supervisão esse número cai para $0,6 \%$ de pessoas com deficiência $(0,2 \%$ têm deficiência física, $0,09 \%$ têm deficiência visual, $0,3 \%$ têm deficiência auditiva e $0,01 \%$ têm deficiência múltipla).

Além de diferenças em relação ao número de pessoas por tipo de deficiência inseridas nas empresas, a
RAIS (MTE, 2012) verificou, no Brasil, a existência também de diferenças referentes à remuneração por tipo de deficiência. As pessoas com deficiência física possuem a maior remuneração entre as pessoas com todos os tipos de deficiência. $\mathrm{O}$ menor rendimento salarial foi observado entre as pessoas com deficiência intelectual.

Analisando as estatísticas expostas, constata-se uma desvantagem profissional de acordo com o tipo de deficiência. Ou seja, a dificuldade de inserção no mercado de trabalho parece estar relacionada não só ao fato de possuir uma deficiência, mas ainda pode ter relação com o tipo de deficiência que a pessoa possui.

Observando os dados estatísticos (Instituto Ethos, 2012; MTE, 2012) e de pesquisas (Carneiro \& Ribeiro, 2008; Gil, 2002; Goulart \& Coimbra, 2008) que indicam uma inserção diferenciada das PcDs no mercado de trabalho, esta pesquisa buscou responder se os gestores avaliam o desempenho da pessoa com deficiência tendo por critério o tipo de deficiência.

\section{MÉTODO}

\section{Participantes}

O estudo foi realizado com 123 gestores de 14 empresas públicas e privadas, de diversos segmentos, com mais de 100 empregados, que possuem trabalhadores com deficiência em seu quadro e estão localizadas no Espírito Santo (ES). O segmento de Prestação de Serviços foi o que concentrou o maior número de empresas participantes $(28,57 \%)$. A maioria das organizações pesquisadas $(64,28 \%)$ existe há mais de 30 anos no mercado, possui mais de 1.000 empregados $(57,14 \%)$ e é de propriedade privada $(64,28 \%)$.

No que se refere à caracterização sociodemográfica dos gestores, 56,9\% deles são do sexo masculino, $68,3 \%$ são casados, $43,9 \%$ têm entre 41 e 50 anos e $56,1 \%$ afirmam praticar a religião católica. O grau de escolaridade predominante é de pessoas que possuem Ensino Superior Completo (36,6\%), seguido por pessoas que possuem Especialização (35,8\%). As entrevistas indicaram que $59,4 \%$ dos gestores têm noções básicas das leis para inclusão das PcDs no mercado de trabalho e $61,9 \%$ dos entrevistados afirmaram possuir informações superficiais, obtidas por meio de jornais e televisão, sobre as PcDs. 
Com relação às pessoas com deficiência geridas pelos respondentes, a maioria dos entrevistados $(71,5 \%)$ gerencia pessoas com deficiência física, sendo que somente $22 \%$ dos gestores da pesquisa não gerenciam pessoas com esse tipo de deficiência. As pessoas com deficiência auditiva são geridas por $38,9 \%$ dos respondentes, seguidas por gestores que gerenciam pessoas com deficiência visual $(16,2 \%)$ e pessoas com deficiência intelectual $(10,5 \%)$. Observou-se um percentual muito baixo de entrevistados que gerem pessoas com deficiência múltipla $(3,3 \%)$.

A maioria dos entrevistados $(56 \%)$ gerencia pessoas com deficiência que ocupam cargos operacionais. Cargos administrativos também são ocupados com frequência $(41,5 \%)$ pelas pessoas com deficiência supervisionadas pelos gestores. Observou-se um percentual baixo $(0,8 \%)$ de participantes que chefiam PcDs em posições gerenciais. Esses dados corroboram os encontrados por Heinski (2004) e Doval (2006), que, em seu estudo, verificou que $81 \%$ das PcDs que trabalhavam nas empresas que pesquisou ocupavam cargos operacionais, sendo que $44 \%$ delas estavam lotadas em atividades administrativas, $25 \%$ em atividades de produção e $12 \%$ de atendimento. Além de as PcDs serem contratadas para cargos cujas atividades a serem executadas são mais simples, como verificado por Tanaka (2007), em todas as classificações de cargos as pessoas com deficiência física têm maior predominância quanto à ocupação das mesmas.

\section{Instrumentos}

Aplicou-se um questionário que visou caracterizar os sujeitos e as empresas participantes. Além disso, foi necessário construir um inventário para avaliar a percepção dos gestores sobre o desempenho das pessoas com deficiência, uma vez que não foi identificado na literatura um instrumento para esse fim, isto é, destinado às pessoas com deficiência, em contextos de trabalho diversos.

O desempenho foi considerado como um fenômeno de natureza multifacetada, que, conforme Coelho Júnior e Borges-Andrade (2011), está associado simultaneamente ao nível individual (querer fazer), à tarefa (saber fazer) e ao contexto de trabalho (poder fazer). Foram operacionalizadas 17 assertivas sobre o desempenho, considerando as discussões de Coelho Júnior e Borges-Andrade (2011), Bendassolli e Mal- vezzi (2013) e Peixoto e Caetano (2013). Essas assertivas (itens) foram encaminhadas para cinco pesquisadores (juízes) que trabalham com a questão do desempenho. Esses juízes avaliaram a pertinência dos itens e sugeriram modificações no inventário (validade de construto). $\mathrm{O}$ inventário foi, então, aplicado em uma amostra pré-teste (análise semântica) composta por oito profissionais que atuavam com pessoas com deficiência no ambiente de trabalho (pessoas com perfil similar aos futuros respondentes da pesquisa), tendo sido realizadas as modificações sugeridas. A versão do inventário utilizada para a presente pesquisa foi composta por 14 questões contemplando aspectos individuais e de resultados, principalmente.

Os itens foram distribuídos em Escala Likert, de escolha forçada, considerando- se a variação de 1 a 6 , sendo o valor $1=$ péssimo e o $6=$ excelente. $\mathrm{O}$ questionário desenvolvido foi denominado Inventário de Percepção do Desempenho no Ambiente de Trabalho (IPDAT).

Ademais, foram realizadas entrevistas semiestruturadas com o objetivo de investigar com maior profundidade as informações coletadas no questionário, de modo a contemplar questões que porventura não constaram nele.

\section{Procedimentos}

$\mathrm{Na}$ análise quantitativa dos dados foram utilizadas as seguintes técnicas estatísticas: a) Estatística descritiva: análise das frequências percentuais das respostas, a fim de descrever o perfil dos gestores participantes da pesquisa e das empresas onde atuam. b) Análise fatorial: identificar a dimensionalidade IPDAT e avaliar evidências de sua confiabilidade (por meio do Teste de Alfa de Cronbach). c) Análise de correspondência: analisar, em relação aos critérios de avaliação de desempenho mais comumente utilizados, se os gestores avaliavam as $\mathrm{PcDs}$ diferentemente, de acordo com o tipo de deficiência que possuíam. A análise de correspondência visa à redução dimensional, permitindo a análise simultânea de um grande número de variáveis, o que a torna indicada para a identificação de relações sistemáticas, de interdependências, entre variáveis (Cunha, 2000; Hair, Anderson, Tatham \& Black, 2005). De acordo com esses autores, a análise de correspondência consiste em uma técnica estatística multivariada, de caráter exploratório, adequada 
para a análise de dados coletados por meio de escala tipo Likert. d) Para a análise das entrevistas, foi utilizada como referência a técnica de análise de conteúdo categorial temática proposta por Bardin (1994).

\section{RESULTADOS}

\section{Análise Fatorial do Inventário de Percepção do Desempenho no Ambiente de Trabalho}

Para a verificação da estrutura dimensional do Inventário de Percepção do Desempenho no Ambiente de Trabalho (IPDAT), utilizou-se do procedimento de Análise Fatorial, buscando sumarizar os dados e identificar as dimensões que compunham os construtos mensurados. Os resultados indicaram a existência de um fator com eigenvalue superior a 1,0, que explica $69 \%$ da variância total. Todos os 14 itens do inventário foram mantidos nas análises, pois apresentaram carga fatorial superior a 0,30 e não apresentaram ambiguidade. $\mathrm{O}$ índice de Kaiser-Meyer-Olkin (KMO = 0,932) e o teste de esfericidade de Bartlett ( $\mathrm{p}<0,001)$ indicaram a fatorabilidade dos dados. $\mathrm{O}$ fator identificado foi denominado 'análise de desempenho'.

Para verificar a confiabilidade do fator, foi utilizado o coeficiente alfa de Cronbach (Tabachnick \& Fidell, 2007), obtendo o valor do coeficiente alfa de 0,965 .

Tabela 1

Análise Fatorial dos Itens do Inventário de Percepção do Desempenho no Ambiente de Trabalho (IPDAT)

35. Qualidade do trabalho (exatidão, frequência de erros, apresentação, ordem e cuidado na realização do trabalho).

36. Produtividade (produção ou quantidade de trabalho realizado).

0,830

37. Capacidade de realização (capacidade de efetivação de idéias e projetos).

0,794

38. Tempo de aprendizagem (tempo destinado à aprendizagem do trabalho).

0,833

39. Iniciativa (identificação de novas alternativas para a realização do trabalho, sugestões para solução de problemas e proposição de melhorias nas atividades desempenhadas).

40. Criatividade (capacidade de propor novas idéias e projetos).

41. Determinação (capacidade de iniciar e concluir um trabalho proposto).

42. Autonomia (capacidade de realizar atividades de forma independente, isto é, sem ajuda constante dos demais).

0,880

43. Comprometimento com os resultados (interesse, dedicação e seriedade na realização dos trabalhos).

0,812

44. Cumprimento das Normas da Organização (assiduidade, pontualidade e atendimento às normas de segurança no trabalho).

45. Aproveitamento dos recursos disponíveis (evita desperdício de tempo, de trabalho e de material).

0,827

46. Capacidade da pessoa com deficiência para lidar com conflitos (capacidade de lidar com situações em que as pessoas tenham posições diferentes sobre determinado assunto, capacidade de lidar com a agressividade das pessoas).

47. Sociabilidade (capacidade da pessoa com deficiência para manter bom relacionamento com os colegas de trabalho e demais grupos dentro da organização; capacidade de negociar e aceitar opiniões diferentes).

48. Cooperação (disposição para colaborar espontaneamente com outras pessoas na realização dos trabaIhos, buscando facilitar o alcance dos resultados). 


\section{A percepção do desempenho das pessoas com deficiência, por tipo de deficiência}

Os resultados em relação à percepção que os gestores possuem frente ao desempenho das pessoas com deficiência, por tipo de deficiência, estão apresentados a seguir (Tabela 2). Esses dados são referentes às questões coletadas por meio do IPDAT. Esses resultados indicam que os gestores veem o desempenho das pessoas com deficiências auditiva e física como positivos. É importante relembrar que a maior parte dos entrevistados gerencia pessoas com deficiência física e esse contato pode interferir na avaliação que fazem em relação a elas. Nenhum gestor marcou os pontos 1 (péssimo) ou 2 (muito ruim) da escala Likert do IPDAT para se referir ao desempenho das pessoas com um desses dois tipos de deficiência.

Tabela 2

Percepção dos Gestores Acerca do Desempenho das PcDs, por Tipo de Deficiência

\begin{tabular}{cccc}
\hline $\begin{array}{c}\text { Tipo de } \\
\text { Deficiência }\end{array}$ & $\begin{array}{c}\text { \% Negativo } \\
(1-2)\end{array}$ & $\begin{array}{c}\text { \% Mediano } \\
(3-4)\end{array}$ & $\begin{array}{c}\text { \% Positivo } \\
(\mathbf{5 - 6 )}\end{array}$ \\
\hline Auditiva & 0 & 45,5 & $\mathbf{5 4 , 5}$ \\
Física & 0 & 38,2 & $\mathbf{6 1 , 8}$ \\
Intelectual & 3,3 & $\mathbf{7 3 , 9}$ & 22,8 \\
Múltipla & 1,6 & $\mathbf{6 8 , 3}$ & 30,1 \\
Visual & 0,8 & $\mathbf{5 5 , 3}$ & 43,9 \\
\hline
\end{tabular}

Fonte: Dados da Pesquisa, 2010.

No entanto, quanto ao desempenho das pessoas com os demais tipos de deficiência, grande parte dos respondentes o avaliou como mediano, ou seja, nem positivo e nem negativo, principalmente em relação ao desempenho das pessoas com deficiência intelectual (73,9\% dos gestores), sendo que essa também foi a categoria de deficiência menos bem avaliada pelos entrevistados (somente 22,8\% dos entrevistados o percebem como positivo). O que pode justificar essa grande quantidade de entrevistados que fazem essa avaliação mediana é o fato de terem dúvidas quanto ao desempenho dessas pessoas e, por isso, têm dificuldade de posicionamento tanto para uma visão positiva quanto para uma percepção negativa do desempenho delas. Essa dúvida pode estar pautada no fato de poucos gestores (somente 12) chefiarem pessoas com esse tipo de deficiência. Assim, o desconhecimento da forma como atuam no ambiente de trabalho pode tendê-los a marcar as alternativas centrais entre os pontos extremos de avaliação.

Com o intuito de verificar se os gestores avaliam diferentemente o desempenho das PcDs por tipo de deficiência, realizou-se análise de correspondência entre as variáveis 'tipos de deficiência' e 'avaliação de desempenho'. A Figura 1 revela que a quantidade de informação explicada pelos dois eixos é bastante elevada, sendo $77,6 \%$ dela explicados pelo eixo 1 e $16,4 \%$ pelo eixo 2 , perfazendo um total de $94 \%$ da informação restituída.

Tomando como base o eixo 1 (explica o maior percentual de variação dos dados), conclui-se que os gestores acreditam que as pessoas com deficiência física (PcDFs) e as pessoas com deficiência auditiva (PcDAs) possuem desempenho semelhante e positivo no ambiente de trabalho e estão associadas aos critérios de desempenho avaliados mais positivamente pelos gestores, cujos pontos representativos dessas variáveis estão proximamente localizados. A deficiência auditiva tem correspondência, principalmente, com os critérios qualidade do trabalho (35), produtividade (36) e autonomia (42). Já as PcDFs têm como principais critérios associados ao seu desempenho a capacidade de realização (37) e o tempo de aprendizagem (38), ambos com os valores melhor avaliados pelos respondentes quando se toma o eixo 1 como referencial de análise. 


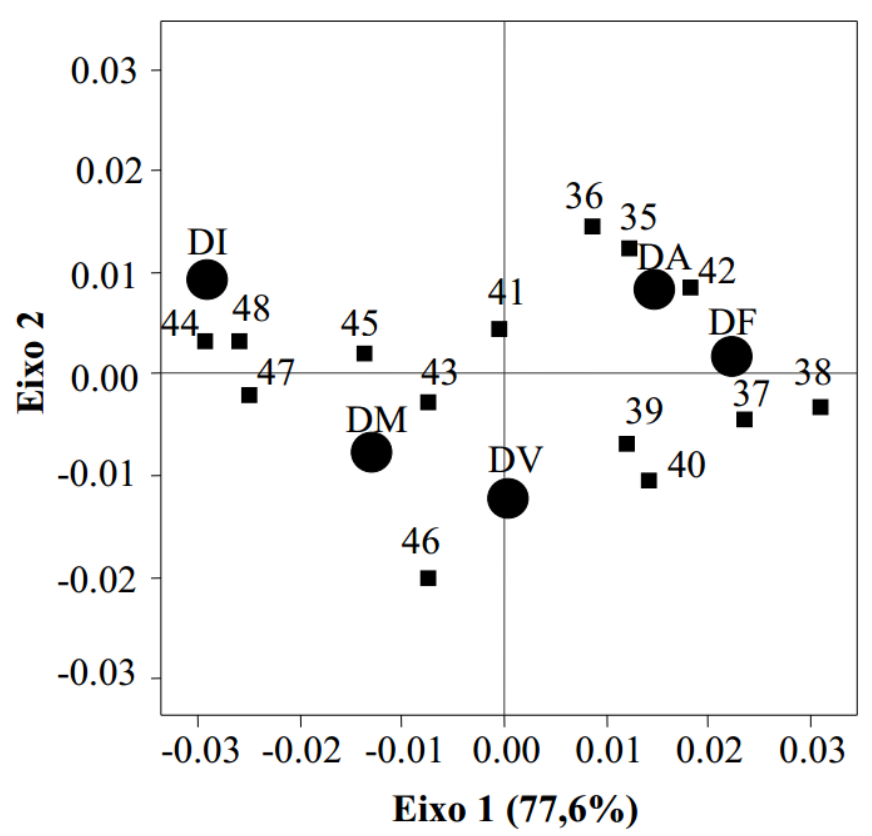

Figura 1. Mapa perceptual das questões sobre desempenho das pessoas com deficiência no trabalho, por tipo de deficiência.

Na figura, tem-se a representação dos tipos de deficiência (deficiência auditiva (DA); deficiência física (DF); deficiência intelectual (DI); deficiência múltipla (DM); deficiência visual (DV)) e a distribuição das questões do IPDAT.

Quando se compara os pontos representativos do desempenho das pessoas com os diversos tipos de deficiência, verifica-se que há uma percepção negativa, por parte dos gestores, quanto ao desempenho das pessoas com deficiência intelectual (PcDIs) e das pessoas com deficiência múltiplas (PcDMs), sendo que os critérios que pesam negativamente na percepção relacionada ao desempenho das PcDs estão associados a essas deficiências, quais sejam: o desempenho das PcDIs tem maior associação com o cumprimento das normas da organização (44), sociabilidade (47) e cooperação (48); enquanto as PcDMs são caracterizadas por terem seu desempenho associado, principalmente, ao comprometimento com os resultados (43) e ao aproveitamento dos recursos disponíveis (45).

Os participantes avaliaram o desempenho das pessoas com deficiência visual (PcDVs) como mediano. Mesmo assim, pode-se afirmar que eles o caracterizam como negativo, pois é nulo em relação ao eixo 1 (seu ponto está localizado no ponto zero desse eixo) e negativo em relação ao eixo 2 . Uma vez que o ponto referente a essa variável está localizado próximo ao centroide, subentende-se que essa avaliação está per- meada por dúvidas quanto à caracterização do desempenho das pessoas com esse tipo de deficiência, bem como se depreende a associação da deficiência visual a critérios de desempenho medianos.

\section{Análise de conteúdo das entrevistas}

As entrevistas realizadas com os gestores abordaram questões abertas que permitiram discorrer sobre as ações que direcionam às $\mathrm{PcDs}$, por tipo de deficiência. As respostas dos gestores às entrevistas foram submetidas à análise de conteúdo, cujo referencial teórico adotado é sugerido por Bardin (1994): as respostas foram agrupadas em categorias de acordo com a similaridade do conteúdo; em seguida, as categorias foram contabilizadas em termos da frequência com que apareceram nas entrevistas.

É importante ressaltar que, na entrevista, os gestores analisaram o desempenho das $\mathrm{PcDs}$ que gerem, isto é, que têm um contato direto, diferentemente do que ocorreu ao responderem aos questionários, nos quais eles avaliaram o desempenho das pessoas com diversos tipos de deficiência, sem necessariamente terem que ter tido contato profissional com elas. 
Em relação à qualidade do desempenho das PcDs geridas pelos gestores, observou-se que as PcDAs, PcDIs e PcDVs tiveram seu desempenho considerado Bom por, respectivamente, $32 \%, 57 \%$ e $43 \%$ dos gestores que as chefiam. Segue, a título de exemplificação, algumas falas representativas: "É bom. Ela [PcDA] não desperdiça tempo com conversas paralelas. O que incomodava no início era o uso do celular, mas conversei com ela e se ajustou. Para mim, ela é uma das que mais produz" (entrevistado 4, gerencia PcDA); e, "Se eu medir o avanço desde que chegaram, eu diria que a pessoa com deficiência intelectual superou, excedeu a expectativa que a gente tinha [...]" (entrevistado 49, gerencia PcDI e PcDV).

Esses resultados indicam que os gestores de PcDIs, PcDAs e PcDVs consideram que estas possuem desempenho positivo, o que contribui para levantar a hipótese de que o contato cotidiano no trabalho com essas pessoas pode contribuir para uma melhor percepção de seus desempenhos. Diferentemente de quando não se atua diretamente com essas pessoas (apenas 16,2\% dos gestores trabalhavam diretamente com PcDVs e 10,5\% com PcDIs).

Já o desempenho das PcDFs foi avaliado como Muito bom/Acima do esperado por $33 \%$ dos gestores que as gerenciam: "É excelente. Excelente mesmo. Dou nota 100. É dedicada" (entrevistado 72, gerencia $\mathrm{PcDF}$ ). Merece nota o fato de que tanto nos resultados quantitativos quanto no qualitativo as PcDFs terem o melhor desempenho considerado entre as pessoas com deficiência. Isso pode ocorrer pelo fato dos gestores participantes, e sua maioria $(71,5 \%)$, gerirem PcDFs. Por outro lado, houve controvérsias quanto à qualidade do desempenho das PcDMs, dos três gestores respondentes que supervisionam o trabalho dessas pessoas, um apontou que é Bom, um disse ser Ruim/Abaixo do esperado e o outro afirmou que é Com restrições.

No que tange à facilidade do gestor em trabalhar com pessoas com algum tipo específico de deficiência foram identificadas cinco categorias, a saber: independe do tipo de deficiência, ou seja, tem facilidade com qualquer tipo; tem facilidade de trabalhar com o(s) tipo(s) de deficiência que trabalha, que já gerencia; possui facilidade de trabalhar com o(s) tipo(s) que não trabalha - neste caso, o gestor trabalha com pessoa com algum tipo de deficiência, mas acredita que teria mais facilidade em trabalhar com pessoas que possuem outro(s) tipo(s) de deficiência; não especificou o tipo de deficiência; e nenhum tipo de deficiência, ou seja, sente dificuldade de trabalhar com pessoas com qualquer tipo de deficiência.

A maior parte dos entrevistados (56\%) afirmou ter facilidade de trabalhar com o tipo de deficiência que já gerencia. Para $32,4 \%$ dos entrevistados a facilidade em trabalhar independe do tipo de deficiência: "Qualquer tipo. São experiências que só me fizeram crescer" (entrevistado 27, gerencia PcDF e PcDV). Um ponto a ser destacado é que a facilidade em se trabalhar com um ou outro tipo de deficiência decorre, de acordo com as falas dos entrevistados, principalmente da experiência que o gestor possui com aquele tipo de deficiência:

Inicialmente, eu achava que era difícil trabalhar com pessoa com deficiência visual, mas, a partir da minha experiência, hoje percebo que é o tipo mais fácil. É 'mole'! Eu já me padronizei nesse tipo de necessidade que ela possui e me adaptei. (entrevistado 39, gerencia PcDI e PcDV)

Como aponta Miller (2002), a convivência com a diversidade pode minimizar os vieses e a discriminação por permitir a emergência de representações não categorizadas. É possível, assim, a personalização do relacionamento, uma vez que a pessoa, até então vista como membro de outro grupo, passa a ser considerada a partir de suas peculiaridades, de suas qualidades únicas.

\section{DISCUSSÃO}

A presente pesquisa permitiu verificar empiricamente uma maior inserção de pessoas com deficiência física e auditiva no mercado de trabalho, em detrimento das pessoas com deficiência intelectual, como corroboram várias outras pesquisas (Bahia, Schommer \& Santos, 2008; Barbosa-Gomes, 2009; Carmo-Ávila, 2011; Carneiro \& Ribeiro, 2008; Goulart \& Coimbra, 2008; Instituto Ethos, 2012). Isso indica que, ao longo dos anos, mesmo aumentando o número de pessoas com deficiência no mercado formal de trabalho, ainda há uma desigualdade dessa inserção de acordo com o tipo de deficiência que a pessoa possui, o que pode estar associado ao estereótipo dessas deficiências. Foi identificado que o desempenho das pessoas com deficiência física é considerado bom e os critérios mais 
associados a esse desempenho são a capacidade de realização e o tempo de aprendizagem. Também a deficiência auditiva tem sido bem avaliada pelos gestores e os critérios associados ao desempenho delas é a qualidade do trabalho, produtividade e autonomia. Por outro lado, as pessoas com deficiência intelectual, por exemplo, têm uma avaliação pior dos gestores, sendo que os critérios associados ao desempenho negativo delas são a falta de cumprimento das normas da organização, menor sociabilidade e cooperação.

É importante sublinhar que a avaliação feita dos diferentes tipos de deficiência pelos gestores, não pressupunha necessariamente que eles estivessem atuando com todas essas pessoas. Nesse sentido, eles avaliaram o desempenho da maioria delas baseados em estereótipos construídos sobre um possível desempenho que elas teriam. Por outro lado, quando os gestores foram interrogados sobre o desempenho das pessoas com deficiência que atuavam diretamente com eles, essa distinção de desempenho em relação às pessoas com deficiência intelectual, visual ou múltipla não se manteve em todos os casos. A avaliação era particularizada e o desempenho era considerado levando-se em conta a realidade vivida e não o estereótipo construído, modificando essa avaliação, em grande parte dos casos, tornando-a mais positiva. Esse resultado reafirma resultados de pesquisa sobre contato intergrupos (Allport, 1954, Pettigrew, 1997; Pettigrew \& Tropp, 2006; Pettigrew, Tropp, Wagner \& Christ, 2011), que asseguram que o contato pode favorecer a mudança de avaliação do comportamento dessas pessoas.

Por meio destes resultados, verifica-se que, quando não se tem convivência profissional com PcD se analisa menos a pessoa em si e suas diversas características pessoais (sexo, escolaridade, grau de deficiência, etc.) e laborais (qualificação e histórico profissional, entre outros) e mais o tipo de deficiência que ela possui, de modo que este se torna um elemento indicativo sobre a possibilidade de a PcD atender aos objetivos inerentes às metas organizacionais. Assim, a categorização feita, que se norteia pelo rótulo - o tipo de deficiência - oculta informações discretas, contempladas nas entrevistas, as quais dão maiores possibilidades de avaliação de suas capacidades laborais. Essa categorização se reflete nas possibilidades de acesso diferenciado das PcDs no mercado de trabalho, conforme indicado na RAIS de 2012 e anos anteriores. Interessa, contudo, sublinhar que a convivência com as PcDs, também identificada em pesquisas internacionais (Miller, 2002; Shannon, Tansey \& Schoen, 2009), modifica a percepção das possibilidades de trabalho das mesmas, principalmente se oferecidas condições adequadas ao seu desempenho.

Outra questão relevante da presente pesquisa foi a possibilidade de construir e identificar características psicométricas válidas para um inventário de avaliação de desempenho que possa ser utilizado em pesquisas. Futuras pesquisas, em regiões diferentes do país, poderão possibilitar uma maior generalização dos resultados da presente investigação.

\section{REFERÊNCIAS}

Allport, G. W. (1954). The nature of prejudice. Cambridge, Mass: Addison-Wesley Pub. Co.

Alves, M. A., \& Galeão-Silva, L. G. (2004). A crítica da gestão da diversidade nas organizações. Revista de Administração de Empresas, 44(3), 20-29. doi: 10.1590/ S0034-75902004000300003

Araujo, J. P., \& Schmidt, A. (2006). A inclusão de pessoas com necessidades especiais no trabalho: A visão de empresas e de instituições educacionais especiais na cidade de Curitiba. Revista Brasileira de Educação Especial, 12(2), 241-254. doi: 10.1590/S1413-65382006000200007

Bahia, M. S., Schommer, P. C., \& Santos, E. M. (2008). Papéis e práticas empresariais para a inserção profissional de pessoas com deficiência: Reflexões a partir de uma experiência. Em Associação Nacional de Pós-Graduação e Pesquisa em Administração (Ed.), Anais do XXXII Encontro Anual da Associação Nacional de Pós-Graduação e Pesquisa em Administração (p. 1-15). Rio de Janeiro: ANPAD.

Barbosa-Gomes, J. F. (2009). Contribuições da categoria trabalho na formação da identidade da pessoa com deficiência (Dissertação de mestrado). Pontifícia Universidade Católica de Minas Gerais, Belo Horizonte.

Bardin, L. (1994). Análise de conteúdo. Lisboa: Edições 70.

Batista, C. A. M. (2004). Inclusão: Construção na diversidade. Belo Horizonte: Armazém de Idéias.

Bendassolli, P. F., \& Malvezzi, S. (2013). Desempenho no trabalho: Definições, modelos teóricos e desafios à gestão. In L. O. Borges, \& L. Mourão (Orgs.), $O$ trabalho $e$ as organizações: Atuações a partir da psicologia (pp. 53-84). Porto Alegre: Artmed.

Bersch, R. (2006). Tecnologia assistiva e educação inclusiva. In Secretaria de Educação Especial (Eds.), Ensaios Pedagógicos (p. 89-94). Brasília: MEC.

Brandão, H. P., \& Guimarães, T. A. (2001). Gestão de competências e gestão de desempenho: Tecnologias dis- 
tintas ou instrumentos de um mesmo construto? RAERevista de Administração de Empresas, 41(1), 8-15. doi: 10.1590/S0034-75902001000100002

Carmo-Ávila, M. R. (2011). Valores organizacionais e concepções de deficiência: A percepção das pessoas com deficiência (Dissertação de mestrado). Universidade Federal de São João del-Rei, São João del-Rei, Minas Gerais. Retirado de http://www.ufsj.edu.br/portal2repositorio/File/ incluir/REGINA.pdf

Carneiro, R., \& Ribeiro, M. A. (2008). A inclusão indesejada: As empresas brasileiras face à Lei de Cotas para pessoas com deficiência. Em: Associação Nacional de PósGraduação e Pesquisa em Administração (Ed.), Anais do XXXII Encontro Anual da Associação Nacional de Pós-Graduação e Pesquisa em Administração (p. 1-15). Rio de Janeiro: ANPAD.

Carvalho-Freitas, M. N. (2007). A inserção de pessoas com deficiência em empresas brasileiras - Um estudo sobre as relações entre concepções de deficiência, condições de trabalho e qualidade de vida no trabalho (Tese de Doutorado). Universidade Federal de Minas Gerais, Belo Horizonte, Minas Gerais.

Carvalho-Freitas, M. N., \& Marques, A. L. (2007). A diversidade através da história: A inserção no trabalho de pessoas com deficiência. Organizações \& Sociedade, 14(41), 57-78.

Carvalho-Freitas, M. N., Souto, J. F., Simas, A. L. B., Costa, N. B., \& Santos, L. M. (2012). Trabalhar com pessoas com deficiência: futuros profissionais têm essa disposição? [CD-ROM]. Em: Associação Nacional de Pós-Graduação e Pesquisa em Administração (Ed.), Anais do XXXVI Encontro Anual da Associação Nacional de Pós-Graduação e Pesquisa em Administração (p. 1-15). Rio de Janeiro: ANPAD.

Cartilha do Censo 2010 (2012). Pessoas com deficiência. Brasília: SDH-PR/SNPD, 2012.

Coelho Júnior, F. A., \& Borges-Andrade, J. E. (2011). Discussão sobre algumas contribuições da modelagem multinível para a investigação de desempenho no trabalho. Psico-USF, 16 (2), 135-142. doi: 10.1590/S141382712011000200002

Cuddy, A. J. C., Fiske, S. T., \& Glick, P. (2007). The BIAS map: Behaviors from intergroup affect and stereotypes. Journal of Personality and Social Psychology, 92(4), 631-648. doi: 10.1037/0022-3514.92.4.631

Cunha, M. V. M., Jr. (2000). Análise multidimensional de dados categóricos: Aplicação das análises de correspondência em marketing e sua integração com técnicas de análise de dados quantitativos. Revista de Administração da Universidade de São Paulo (RAUSP), 35(1), 32-50.

Decreto n. 3.298, de 20 de dezembro de 1999 (1999). Regulamenta a política nacional para a integração da pessoa portadora de deficiência, consolida as normas de proteção, e dá outras providências. Brasília: Presidência da República.
Doval, J. L. M. (2006). Inclusão de pessoas portadoras de deficiência no mercado de trabalho: desafios e tendências (Dissertação de Mestrado). Universidade Federal do Rio Grande do Sul, Porto Alegre, Rio Grande do Sul.

Fleury, A, \& Fleury. M. T. L. (2001). Estratégias empresariais e formação de competências: Um quebra-cabeça caleidoscópico da indústria brasileira. São Paulo: Atlas.

Gil, M. (Coord.). (2002). O que as empresas podem fazer pela inclusão das pessoas com deficiência. São Paulo: Instituto Ethos.

Goulart, Í. B., \& Coimbra, C. E. P. (2008). Inserção de pessoas com deficiência numa empresa de reflorestamento: um estudo de caso. Em: Associação Nacional de Pós-Graduação e Pesquisa em Administração (Ed.), Anais do XXXII Encontro Anual da Associação Nacional de Pós-Graduação e Pesquisa em Administração (p. 115). Rio de Janeiro: ANPAD.

Hair, J. F., Jr., Anderson, R. E., Tatham, R. L., \& Black, W. C. (2005). Análise multivariada de dados (A. S. Sant'Anna, \& A. Chaves Neto, Trad.) (5 ed.). Porto Alegre: Bookman.

Heinski, R. M. M. S. (2004). Um estudo sobre a inclusão da pessoa portadora de deficiência no mercado de trabalho [CD-ROM]. Em: Associação Nacional de PósGraduação e Pesquisa em Administração (Ed.), Anais do XXIII Encontro Anual da Associação Nacional de PósGraduação e Pesquisa em Administração (p. 1-15). Curitiba: ANPAD.

Instituto Brasileiro de Geografia e Estatística (2000). Censo 2010. Retirado de http://www.sidra.ibge.gov.br

Instituto Ethos. (2012). Perfil social, racial e de gênero das 500 maiores empresas do Brasil e suas ações afirmativas - Pesquisa 2010. São Paulo: Ethos.

Jones, M. K., Latreille, P. L., \& Sloane, P. J. (2006). Disability, gender, and the British labour market. Oxford Economic Papers, 58(3), 407-449. doi: 10.1093/oep/gpl 004

Lei n. 8.213, de 24 de julho de 1991 (1991). Dispõe sobre os planos de benefícios da Previdência Social e dá outras providências.

Miller, N. (2002). Personalization and the promise of contact theory. Journal of Social Issues, 58(2), 387-410. doi: 10.1111/1540-4560.00267

Ministério do Trabalho e Emprego (2012). Mapa do emprego formal. Retirado de http://portal.mte.gov.br/portalmte/rais/

Nascimento, L. C., Damasceno, G. J. B., \& Assis, L. J. (2008). Mercado de trabalho para as pessoas com deficiência em Betim/MG. Em: Associação Nacional de Pós-Graduação e Pesquisa em Administração (Ed.), Anais do XXXII Encontro Anual da Associação Nacional de Pós-Graduação e Pesquisa em Administração (p. 1-15). Rio de Janeiro: ANPAD.

Nohara, J. J., Acevedo, C. R., \& Fiametti, M. (2009). A vida no trabalho: As representações sociais das pessoas com deficiências. Em: M. N. Carvalho-Freitas, \& A. L. 
Marques (Orgs.). Trabalho e pessoas com deficiência: Pesquisas, práticas e instrumentos de diagnóstico (pp. 71-88). Curitiba: Juruá.

Oliveira, E. R. (2008). Ações de inclusão de trabalhadores com deficiência em empresas de diferentes ramos de atividades (Dissertação de Mestrado). Universidade Presbiteriana Mackenzie, São Paulo, São Paulo.

Organização Internacional do Trabalho. (2009). Disability and work. Retirado de http://www.ilo.org/public/english/ employment/skills/disability/index.htm

Peixoto, A. L. A., \& Caetano, A. (2013). Avaliação de desempenho. Em: L. O. Borges, \& L. Mourão (Orgs.). $O$ trabalho e as organizações: Atuações a partir da Psicologia (pp. 528-555). Porto Alegre: Artmed.

Pettigrew, T. F. (1997). Generalized intergroup contact effects on prejudice. Personality and Social Psychology Bulletin, 23(2), 173-185. doi: 10.1177/0146167297232006

Pettigrew, T. F., \& Tropp, L. R. (2006). A meta-analytic test of intergroup contact theory. Journal of Personality and Social Psychology, 90(5), 751-783. doi: 10.1037/ 0022-3514.90.5.751

Pettigrew, T. F., Tropp, L. R., Wagner, U., \& Christ, O. (2011). Recent advances in intergroup contact theory. International Journal of Intercultural Relations, 35(3), 271-280. doi: 10.1016/j.ijintrel.2011.03.001

Ribeiro, M. A., \& Ribeiro, F. (2009). Gestão organizacional da diversidade: Estudo de caso de um programa de inclusão de pessoas com deficiência. Em: M. N. Carvalho-Freitas \& A. L. Marques (Orgs.), Trabalho e pessoas com deficiência: Pesquisas, práticas e instrumentos de diagnóstico (pp. 122-141). Curitiba: Juruá.

Sassaki, R. K. (1999). Inclusão (3 ${ }^{\mathrm{a}}$ ed.). Rio de Janeiro: WVA.

Sassaki, R. K. (2005). Atualizações semânticas na inclusão de pessoas: deficiência mental ou intelectual? Doença ou transtorno mental? Revista Nacional de Reabilitação, 43(9), 9-10.

Shannon, C. D., Tansey, T. N., \& Schoen, B. (2009). The effect of contact, context, and social power on under- graduate attitudes toward persons with disabilities. Journal of Rehabilitation, 75(4), 11-18.

Stone-Romero, E. F., Stone, D. L., \& Lukaszewski, K. (2006). The influence of disability on role-taking in organizations. Em: A. M Konrad, P. Prasad, \& J. K. Pringle (Orgs.). Handbook of workplace diversity (pp. 401430). Thousand Oaks, CA: Sage.

Tabachnick, G. G., \& Fidell, L. S. (2007). Experimental designs using ANOVA. Belmont, CA: Duxbury.

Tanaka, E. D. O. (2007). O desenvolvimento de uma escala de atitudes sociais em relação ao trabalho da pessoa com deficiência (Tese de Doutorado), Universidade Estadual Paulista Júlio de Mesquita Filho, Marília, São Paulo.

Tanaka, E. D. O., \& Manzini, J. E. (2005). O que os empregadores pensam sobre o trabalho da pessoa com deficiência? Revista Brasileira de Educação Especial, 11(2), 273-294. doi: 10.1590/S1413-65382005000200008

Trice, H., \& Beyer, J. (1993). The cultures of work organizations. Englewood Cliffs, NJ: Prentice Hall.

Wilson-Kovacs, D. Ryan, M, Haslam, A., \& Rabinovich, A. (2008). "Just because you can get a wheelchair in the building doesn't necessarily mean that you can still participate": Barriers to the career advancement of disabled professionals. Disability \& Society, 23(7), 705-717. doi: 10.1080/09687590802469198

Zolna, J. S., Sanford, J., Sabata, D., \& Goldthwaite, J. (2007). Review of accommodation strategies in the workplace for persons with mobility and dexterity impairments: Application to criteria for universal design. Technology and Disability, 19(4), 189-198.

\section{Notas:}

1 O termo deficiência intelectual é mais apropriado por referir-se ao funcionamento especificamente do intelecto, e não da mente como um todo. Busca-se, dessa forma, minimizar a associação errônea com as doenças mentais (Sassaki, 2005).

2 Tecnologia assistiva é um conceito recente e em processo de construção. Compreende todo o arsenal de recursos que venham favorecer uma vida mais independente e produtiva para pessoas com deficiência no contexto social geral (Bersch, 2006).

Agradecemos ao CNPq pelo apoio ao financiamento da pesquisa. 